\title{
Considering Angle Selection When Using Ultrasound Electrode Displacement Elastography to Evaluate Radiofrequency Ablation of Tissues
}

\author{
Jingjing Xia, ${ }^{1}$ Qiang Li, ${ }^{1}$ Pin-Yu Chen, ${ }^{2}$ Zhuhuang Zhou, ${ }^{3}$ Chiao-Yin Wang, ${ }^{2,4}$ \\ Hao-Li Liu, ${ }^{5}$ Jianfu Teng, ${ }^{1}$ and Po-Hsiang Tsui ${ }^{2,6}$ \\ ${ }^{1}$ School of Electronic Information Engineering, Tianjin University, Tianjin 300072, China \\ ${ }^{2}$ Department of Medical Imaging and Radiological Sciences, College of Medicine, Chang Gung University, \\ 259 Wen-Hwa 1st Road, Kwei-Shan, Taoyuan, Taoyuan County 33302, Taiwan \\ ${ }^{3}$ Biomedical Engineering Center, College of Life Science and Bioengineering, Beijing University of Technology, Beijing 100124, China \\ ${ }^{4}$ Graduate Institute of Clinical Medical Sciences, College of Medicine, Chang Gung University, Taoyuan 33302, Taiwan \\ ${ }^{5}$ Department of Electrical Engineering, Chang Gung University, Taoyuan 33302, Taiwan \\ ${ }^{6}$ Institute for Radiological Research, Chang Gung Memorial Hospital at Linkou, Chang Gung University, Taoyuan 33302, Taiwan
}

Correspondence should be addressed to Po-Hsiang Tsui; tsuiph@mail.cgu.edu.tw

Received 19 March 2014; Accepted 7 May 2014; Published 27 May 2014

Academic Editor: Tobias De Zordo

Copyright ( $\odot 2014$ Jingjing Xia et al. This is an open access article distributed under the Creative Commons Attribution License, which permits unrestricted use, distribution, and reproduction in any medium, provided the original work is properly cited.

Percutaneous radiofrequency ablation (RFA) is a minimally invasive treatment to thermally destroy tumors. Ultrasound-based electrode-displacement elastography is an emerging technique for evaluating the region of RFA-induced lesions. The angle between the imaging probe and the RFA electrode can influence electrode-displacement elastography when visualizing the ablation zone. We explored the angle effect on electrode-displacement elastography to measure the ablation zone. Phantoms embedded with meatballs were fabricated and then ablated using an RFA system to simulate RFA-induced lesions. For each phantom, a commercial ultrasound scanner with a $7.5 \mathrm{MHz}$ linear probe was used to acquire raw image data at different angles, ranging from $30^{\circ}$ to $90^{\circ}$ at increments of $10^{\circ}$, to construct electrode-displacement images and facilitate comparisons with tissue section images. The results revealed that the ablation regions detected using electrode-displacement elastography were highly correlated with those from tissue section images when the angle was between $30^{\circ}$ and $60^{\circ}$. However, the boundaries of lesions were difficult to distinguish, when the angle was larger than $60^{\circ}$. The experimental findings suggest that angle selection should be considered to achieve reliable electrode-displacement elastography to describe ablation zones.

\section{Introduction}

Liver cancer is a major public health problem worldwide, and, based on data compiled by the National Center for Health Statistics in 2013 [1], the mortality rate of liver cancer has steadily increased over the previous 3 decades. Several alternative therapeutic methods are available for hepatic tumors in addition to chemotherapy, such as surgical resection or liver transplants [2-4]. Because patients with poor liver function or distributed tumors are unsuited to undergoing surgical resection and liver transplant, radiofrequency ablation (RFA) is used as a minimal invasive strategy for liver tumor treatment. RFA is one of the most popular choices for hepatic tumor treatments $[5,6]$.

During RFA, a radiofrequency (RF) needle electrode is inserted into the tumor to deliver a strong alternating electrical current. This current agitates ions and produces a temperature increase that induces the coagulation necrosis of tissues surrounding the electrode. Paramount to the success of RFA therapy is that the ablation region adequately covers the entire tumor to ensure a complete destruction of cancer cells. Thus, developing imaging modalities that accurately portray the ablation zones is crucial [7]. Ultrasound is the most frequently used modality to monitor 


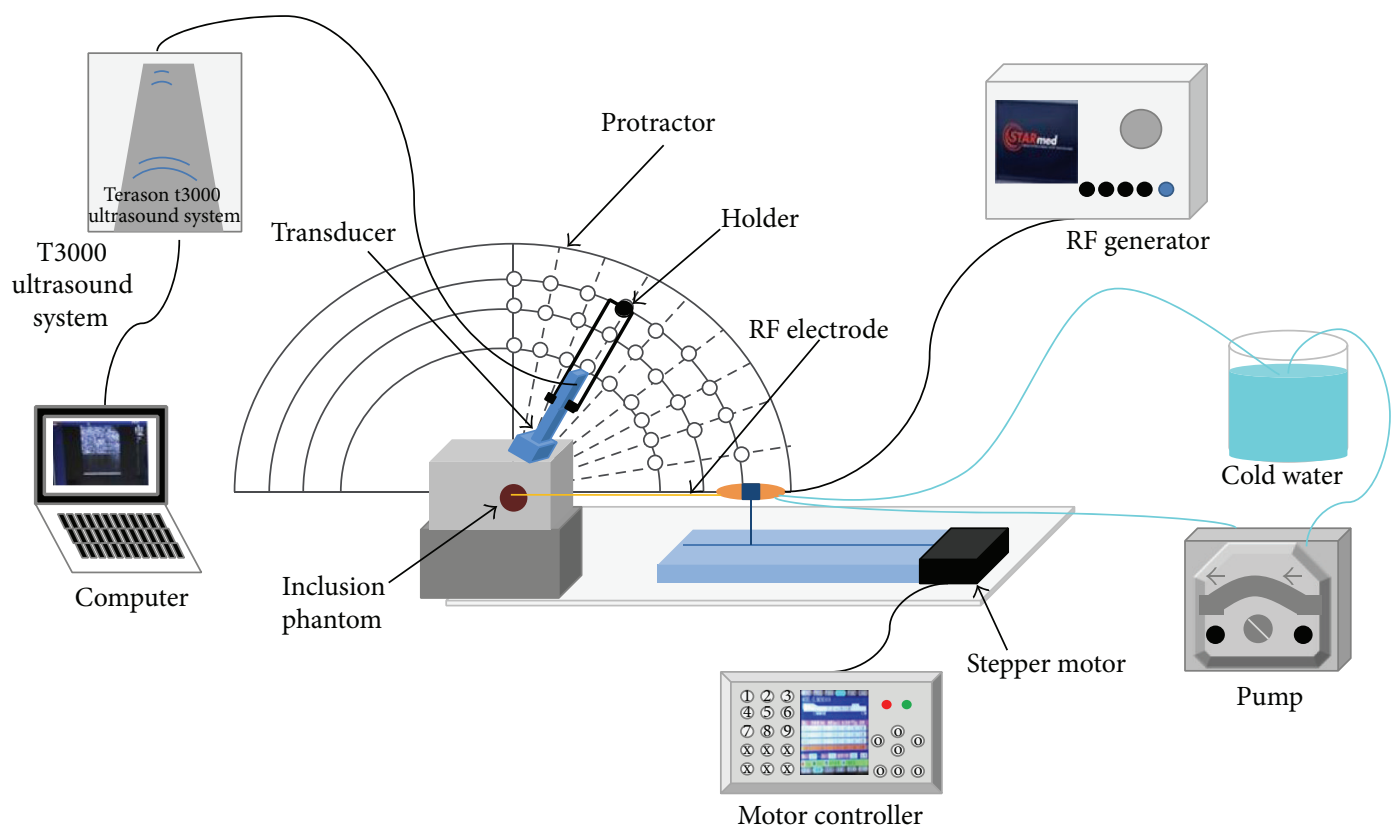

Figure 1: Schematic illustration of the experimental setup for exploring the angle effect on using electrode-displacement elastography to monitor RFA.

RFA because of its portability, low cost, real-time capability, and compatibility with other medical equipment. RFAinduced necrosis tissue has a greater stiffness compared with the background untreated tissues [8-11]. Consequently, ultrasound elastography, a functional ultrasound image that reflects tissue stiffness, might provide reliable, high-contrast images for monitoring the ablation region $[12,13]$. Ultrasound elastography can be classified into 3 types: (1) those that apply a quasistatic compression and estimate the resulting components of the strain tensor [12-18]; (2) those that apply low-frequency vibration with ultrasound Doppler detection of velocities of perturbed reflectors [19-21]; and (3) those that use acoustic-radiation force $[8,9,22]$.

In the current clinical diagnosis, the type I elastography previously mentioned (i.e., strain imaging) is widely used because of its popularity and compatibility with the conventional pulse-echo ultrasound system. However, when applying the ultrasound strain image to describe the ablation zone, using the transducer to compress tissues (external compression) might be difficult in producing internal strain in liver tissue in vivo. To address this concern, Varghese et al. $[8,9,23,24]$ proposed adopting perturbations of the $\mathrm{RF}$ electrode as the mechanical stimulus to induce tissue displacements for elastography. The incremental displacements applied to the electrode provide localized compression in the thermal lesion [25]. Nightingale et al. [26] also employed localized compression in using acoustic-radiation force to introduce perturbations in the targeted tissue region. Bharat et al. $[23,24,27-30]$ performed both phantom and in vivo animal experiments to validate that electrode-displacement elastography is capable of providing high-contrast images, using widely available commercial ultrasound systems that can be used to assess the extent of thermal ablation zones.
The aforementioned studies placed the imaging probe parallel to the RF electrode when performing electrodedisplacement strain imaging for monitoring RFA to measure the maximum strain of the tissue for imaging (the angle between the imaging transducer and the RF electrode should be as small as possible). However, such an arrangement might limit the clinical applications of electrode-displacement strain imaging. Tumors can form at any depth of liver tissue and thus might be outside the effective range of electrodedisplacement strain imaging based on the suggested minimum angle. Monitoring the RFA of tumors at various depths and locations might necessitate using various angle arrangements for electrode-displacement strain imaging in future clinical applications. Establishing a multiangle-based electrode-displacement strain imaging is a long-term goal. Therefore, the effects of angles on electrode-displacement strain imaging must be clarified. Because of the absence of research on the effects of angles on electrode-displacement elastography, we investigated the effects of angles on using electrode-displacement strain imaging to monitor RFA.

\section{Materials and Methods}

2.1. Experimental Setup. The experimental setup (Figure 1) consisted of 4 major parts: a clinical RFA system, a commercial ultrasound imaging system, a protractor, and a stepper motor system. The RFA system (Model VIVA RF Generator, STARmed Co. Ltd., Goyang, Gyeonggi, Republic of Korea) included a cool-tip RF electrode (Model 17-20V1540, STARmed Co.) and an RF generator for providing an adjustable RF output power ranging from 0 to $200 \mathrm{~W}$, with an operation frequency of $480 \mathrm{kHz}$. The RFA system also included a peristaltic pump, which was used to deliver 


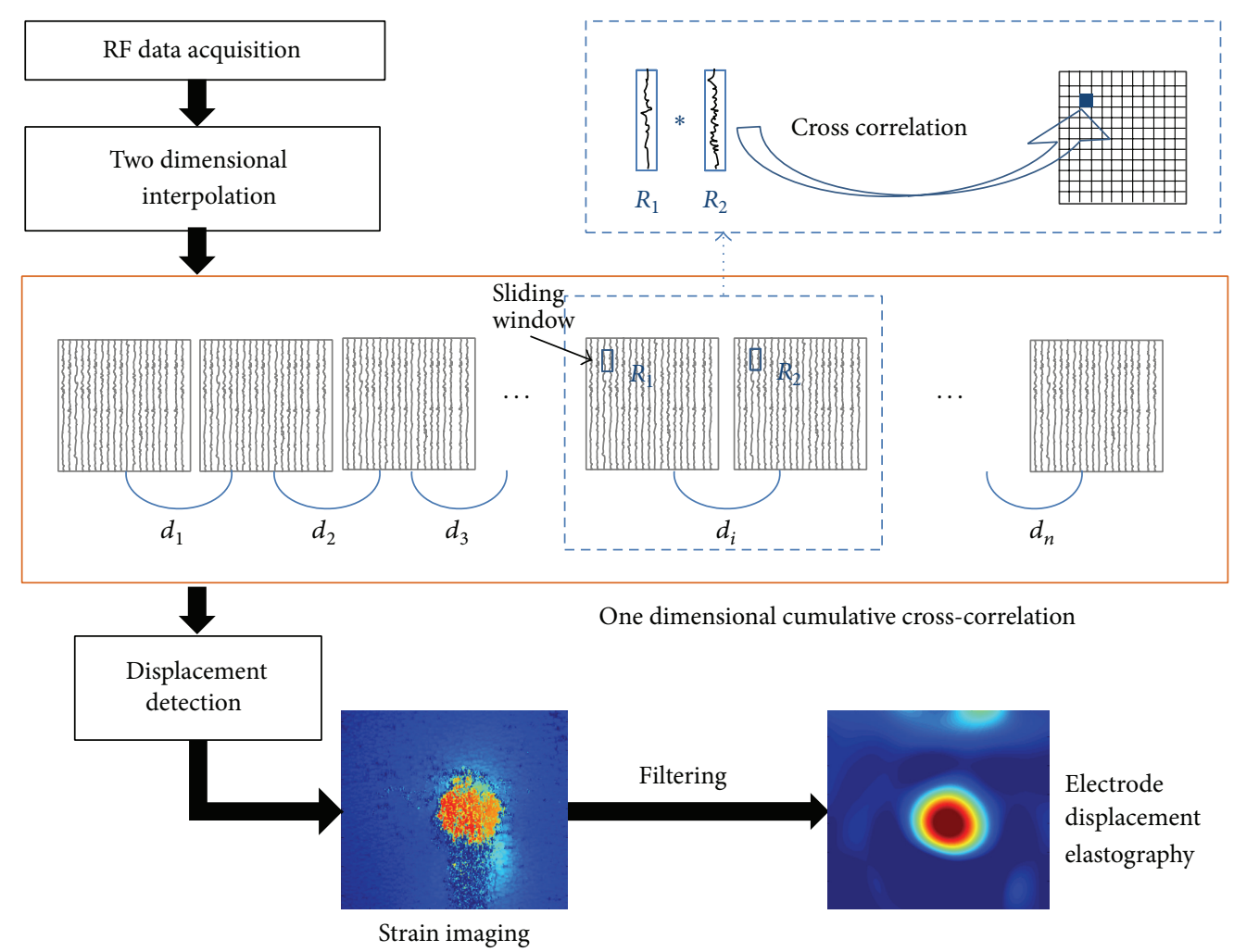

FIgURE 2: Flow chart of the algorithm for constructing the electrode-displacement elastography.

a constant flow of cold saline solution with a mixture of ice to the electrode tip for preventing the electrode from overheating, resulting in tissue carbonization. The ultrasound imaging system (Model 3000, Terason, Burlington, MA, USA) was equipped with a $7.5 \mathrm{MHz}$ linear array transducer (Model 12L5V, Terason), which was used to monitor RFA and acquire raw backscattered RF data at a sampling rate of $37.5 \mathrm{MHz}$ for subsequent electrode-displacement imaging. The protractor was used to calibrate the angle between the imaging transducer and the RF electrode from $30^{\circ}$ to $90^{\circ}$ in increments of $10^{\circ}$. The stepper motor system (Model ELS2XE003-KP, Oriental Motor, Tokyo, Japan) with a selfmade electrode holder was used to move the RF electrode back and forth for inducing displacement of tissue samples.

2.2. Material Preparation and Experimental Procedure. In order to explore the angle effect on electrode-displacement elastography, we referred to a previous study [23] to construct agar phantoms embedded with meatball inclusions $(n=$ 14). In practice, an intermediate transition zone will appear between the unablated and the RFA-induced necrotic tissues, making it difficult to accurately identify the ablation region. For this reason, we used phantoms with meatballs instead of whole tissue samples in the experiments to provide a standard ablation size for comparison (necrosis following RFA only occurs in part of the inclusion). The diameter of the embedded inclusions ranged from 11.09 to $19.12 \mathrm{~mm}$, with a mean and standard deviation of $140.19 \pm 51.48 \mathrm{~mm}^{2}$. After embedding the meatball in the agar phantom, RF electrode was inserted into the meatball inclusion to ablate the tissue. We have confirmed that the settings (ablation time and power, described in the next paragraph) used for RFA allow the ablation of the meatball inclusion completely. In the phantom, we added graphite powder with diameter $<20 \mu \mathrm{m}$ (Model 282863, Sigma-Aldrich, St. Louis, MO, USA) to simulate scatterers and image speckle in the background. The previous study [23] revealed that the agar phantom embedded with the ablated inclusion can produce the difference of the stiffness between the phantom background and the ablation zone for testing the electrode-displacement elastography.

For each inclusion phantom, RFA was performed at the default automatic mode, which started at 50 and automatically increased by $10 \mathrm{~W} / \mathrm{min}$ until the first RF pulse paused because of high tissue impedance before generating a sequence of RF pulses as a time function. The treatment time for the automatic mode was $12 \mathrm{~min}$. Following the ablation, the inclusion phantom cooled for $12 \mathrm{~min}$. The stepper motor system subsequently controlled the RF electrode to produce tissue displacements of $1 \mathrm{~mm}$. An image frame was acquired when the RF electrode moved forward $0.1 \mathrm{~mm}$. In the range of $1 \mathrm{~mm}$, a total of 10 image frames were acquired from the ultrasound system for electrode-displacement imaging. These measurements were repeated for angles from $30^{\circ}$ to $90^{\circ}$.

\subsection{Algorithm for Electrode-Displacement Elastography.} Figure 2 shows the flow chart of the algorithm for electrodedisplacement imaging. One-dimensional cross-correlation analysis of 2 consecutively acquired images of RF data 
TABLE 1: Comparisons between the inclusion sizes and the ablation zones detected by electrode displacement strain imaging. The correlation coefficient decreases with increasing the angle between the transducer and the RF electrode. The correlation coefficient is smaller than 0.9 when the angle is larger than $60^{\circ}$. Data are expressed by mean \pm standard deviation.

\begin{tabular}{lcccc}
\hline Angle & $\begin{array}{c}\text { Meatball size } \\
\text { (inclusion phantom) }\end{array}$ & $\begin{array}{c}\text { Ablation size } \\
\text { (electrode displacement strain image) }\end{array}$ & Error (\%) & Correlation coefficient \\
\hline $30^{\circ}$ & $140.19 \pm 51.48$ & $152.87 \pm 51.36$ & $6.65 \pm 1.51$ & 0.9841 \\
$40^{\circ}$ & $140.19 \pm 51.48$ & $148.39 \pm 46.75$ & $10.13 \pm 2.07$ & 0.9544 \\
$50^{\circ}$ & $140.19 \pm 51.48$ & $139.73 \pm 44.60$ & $11.00 \pm 1.86$ & 0.9438 \\
$60^{\circ}$ & $140.19 \pm 51.48$ & $136.64 \pm 59.77$ & $67.02 \pm 2.71$ & 0.9331 \\
$70^{\circ}$ & $140.19 \pm 51.48$ & $41.12 \pm 41.29$ & $89.92 \pm 3.33$ & 0.0361 \\
$80^{\circ}$ & $140.19 \pm 51.48$ & $12.55 \pm 18.80$ & 100 & 0.0800 \\
$90^{\circ}$ & $140.19 \pm 51.48$ & N/A & 0 \\
\hline
\end{tabular}

following interpolation was performed using a $3 \mathrm{~mm}$ long window with an overlap ratio of $75 \%$ to obtain the tissuedisplacement image. The strain images were obtained from the axial differentiation of the displacement image. To remove noise and smooth images, we applied a $3 \times 3$ median filter and an 8-order 2-dimensional Butterworth low-pass filter at the cutoff frequency of $5 \mathrm{MHz}$ to process the strain image.

2.4. Data Comparison. For each inclusion phantom, the size of the meatball was measured as the ground truth of the ablation zone. We used the Image free image analysis software to outline the ablation zone detected in the electrodedisplacement image. At first, a square region of interest only containing the ablation zone detected in the image was used as a subimage. Then, we used the ImageJ software to adjust the threshold color of the subimage, until the contour of the ablation zone was well detected. By calibration using the scale of the electrode-displacement image, ImageJ software automatically measured the size of the segmented ablation area. The ablation sizes obtained from the sample measurements and the electrode-displacement image were compared using linear regression in the form of $y=y_{0}+a x$ to calculate the correlation coefficient, thereby determining the angle effect on using electrode-displacement imaging to visualize the ablation zones.

\section{Results}

Figure 3 shows B-mode images of the inclusion phantom obtained at various angles ranging from $30^{\circ}$ to $90^{\circ}$ after RFA. The conventional B-mode image indicated no ability to identify the change in tissue stiffness after RFA. Figures 4 and 5 present the corresponding electrode-displacement and strain images. The ablation zone (the inclusion region) was clearly described using electrode-displacement strain imaging, when the angle between the transducer and the electrode was smaller than $60^{\circ}$. However, when the angle exceeded $60^{\circ}$, the ablation zone detected in the electrode-displacement elastography gradually disappeared. This result indicated that using electrode-displacement elastography to monitor RFA is highly dependent on the angle between the transducer and the RF electrode.
To further confirm the experimental findings, we explored the relationship between the inclusion sizes and the ablation zones detected using the electrode-displacement strain image (Figure 6). Table 1 summarizes the comparison results. When the angles were smaller than $60^{\circ}$, the correlation coefficients obtained from the linear regression were larger than 0.9 and the error reduced to less than $15 \%$. However, the correlation coefficient reduced to less than 0.05 and the error increased when the angle was larger than $60^{\circ}$.

\section{Discussion}

4.1. The Significance of This Study. Electrode-displacement strain imaging involves using the RF electrode as a displacement device, which provides localized compression in the region of interest. This displacement mechanism offers the possibility of in vivo implementation because it is difficult to produce tissue strain through conventional ultrasound elastography using external compression techniques. Before using the electrode-displacement strain image as a reliable clinical tool for applications, certain issues require further investigation. One critical issue is the effect of the imaging angle between the ultrasound transducer and the RF electrode on the performance of electrode-displacement strain imaging in monitoring RFA. We clarified the angle issue and suggested a prerequisite angle $<60^{\circ}$ for a successful formation of electrode-displacement strain imaging.

\subsection{Physical Meaning of Electrode-Displacement Imaging.} During the RFA procedure, the temperature in the tissue typically reaches boiling temperature. The ablation zone behaves similarly to a hyperechoic region in the ultrasound image because of bubble formation under such high-temperature ablation. Previous studies have observed that bubbles insonified through ultrasound with sufficient intensity and duration can emit various subharmonic components [31] and other low frequency emissions $[32,33]$. Based on this viewpoint, Winkler and Adam [34] proposed using acoustic emissions at low frequencies from the RFA-induced bubbles for assessing RFA monitoring.

However, by decreasing the tissue temperature following the RFA procedure, the dissolved bubbles cause the hyperechoic region to disappear, and the ablation zone typically 


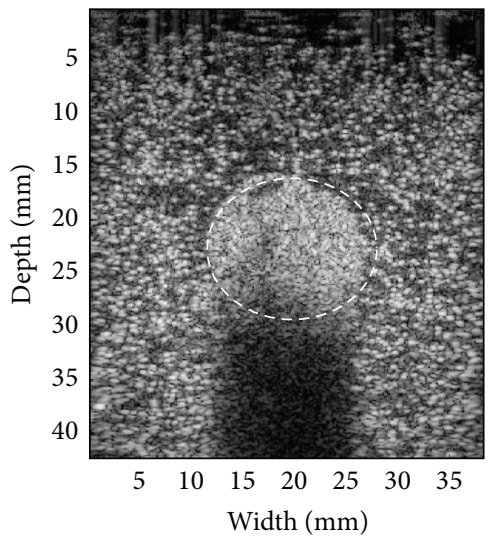

(a)

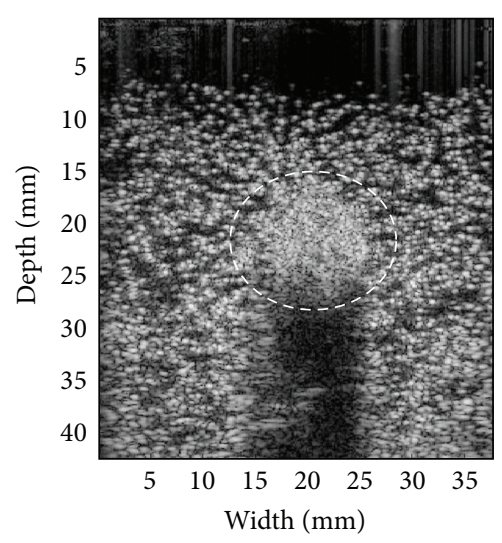

(d)

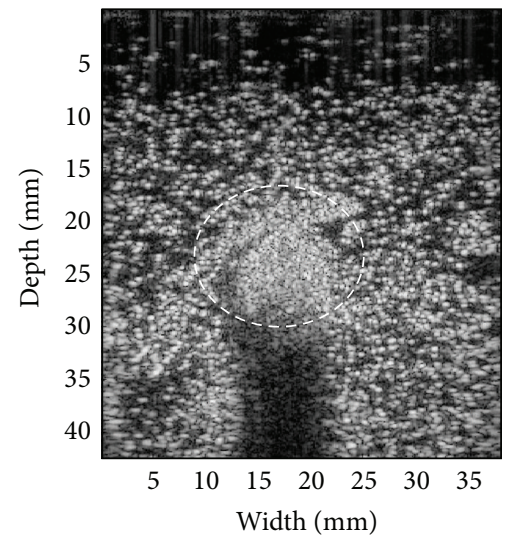

(b)

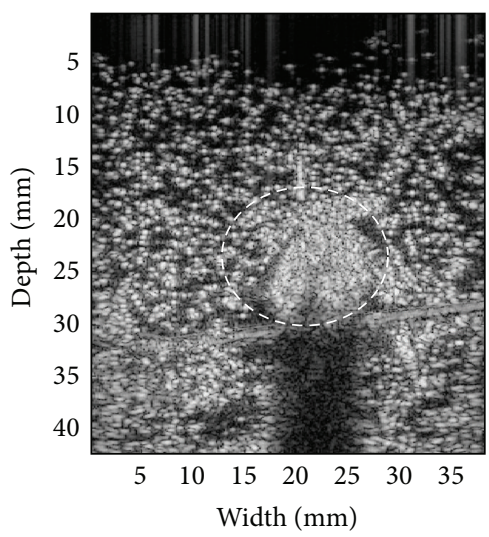

(e)

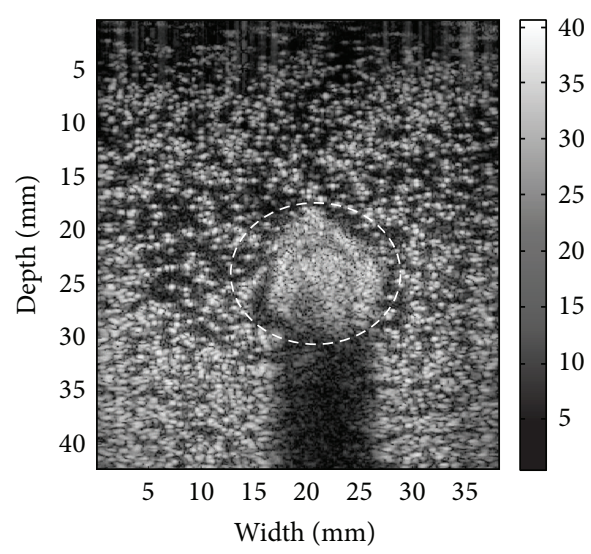

(c)

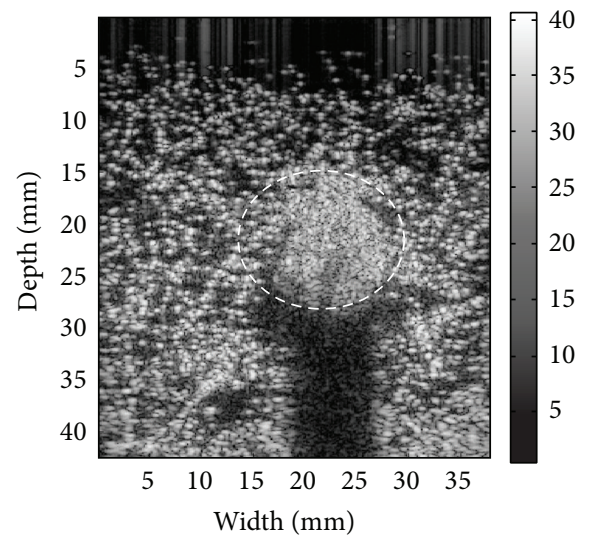

(f)

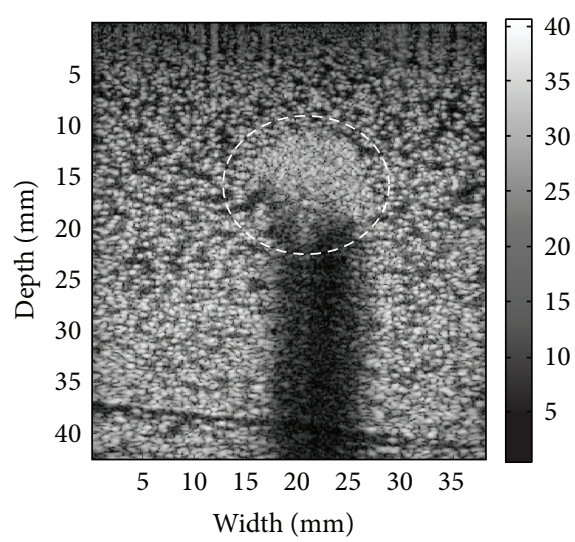

(g)

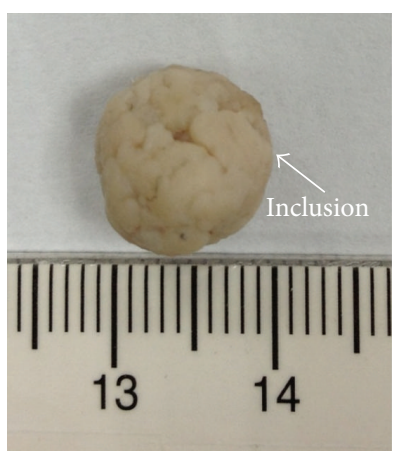

(h)

Figure 3: B-mode images obtained at different angles: (a) angle $=30^{\circ}$, (b) angle $=40^{\circ}$, (c) angle $=50^{\circ}$, (d) angle $=60^{\circ}$, (e) angle $=70^{\circ}$, (f) angle $=80^{\circ}$, and (g) angle $=90^{\circ}$; (h) embedded meatball in the inclusion phantom.

manifests as a mixed echogenicity zone in the B-mode image. Tissue stiffness subsequently becomes crucial for evaluating the performance of RFA treatment. RFA-induced necrosis zones are considerably stiffer than untreated regions. In principle, the displacement detected in ablation zones should be small compared with that of untreated tissues. Although previous studies have observed that the ablation zone produces small tissue displacement $[8,9]$, our experiment results revealed the opposite phenomenon. The results of electrodedisplacement imaging (Figure 4) showed that the ablation zone exhibited large displacement. We assumed that the tissue displacement might rely on the adhesion force of the ablation tissue to the RF electrode. In our experiments, RFA was operated at the default mode of $50 \mathrm{~W}$ to produce an extremely high temperature. We observed that the RF electrode strongly adhered to the necrotic tissue after RFA 


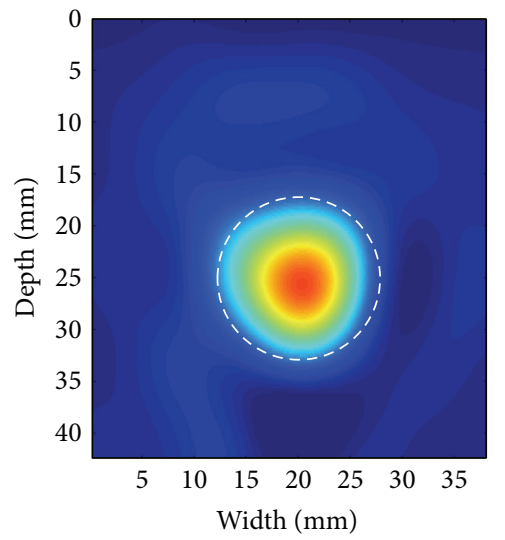

(a)

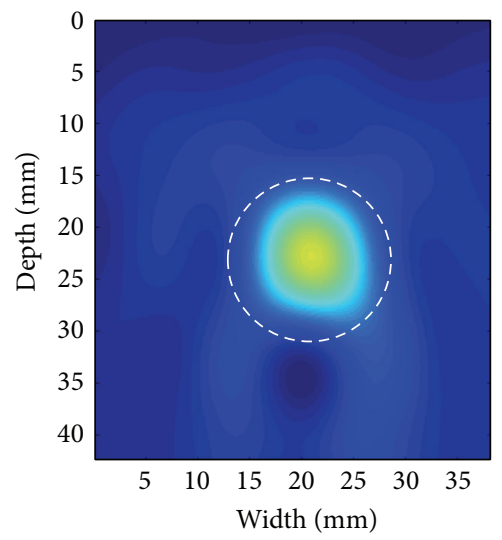

(d)

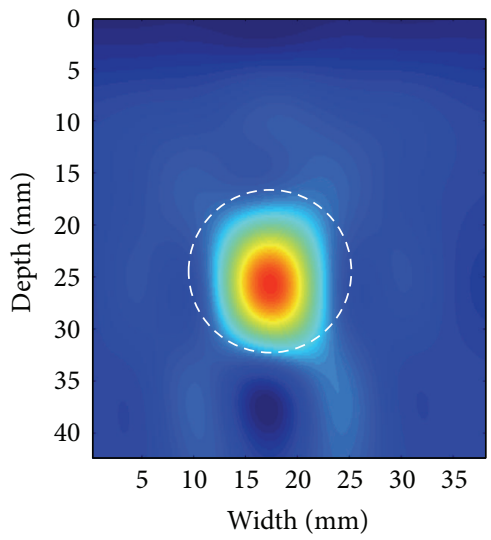

(b)

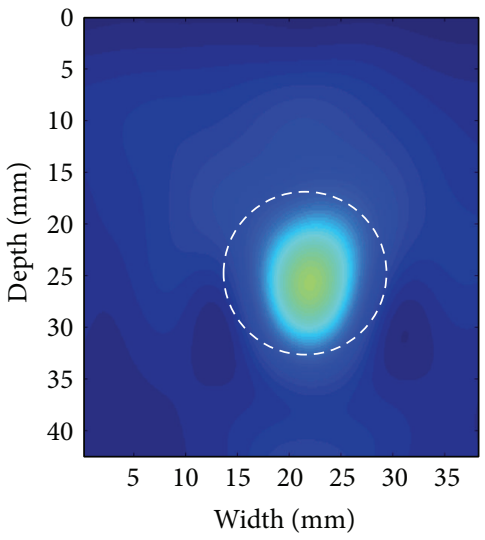

(e)

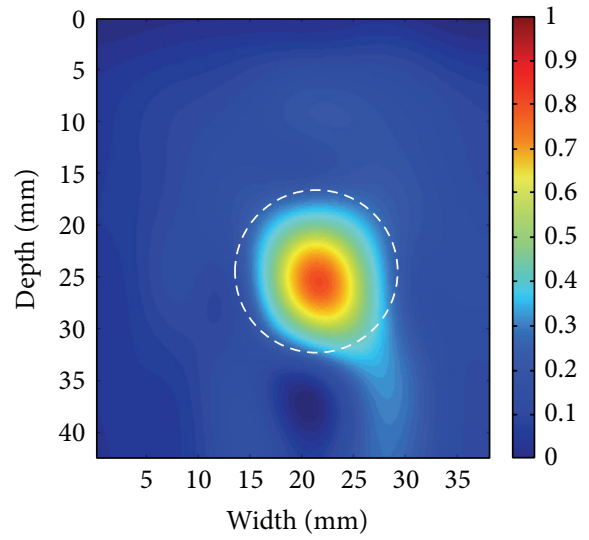

(c)

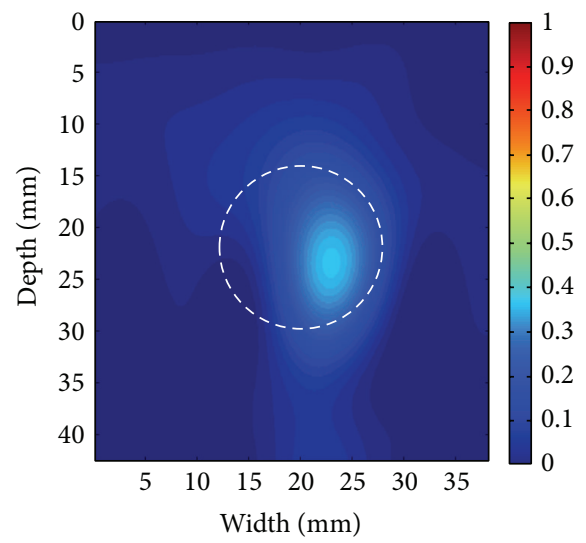

(f)

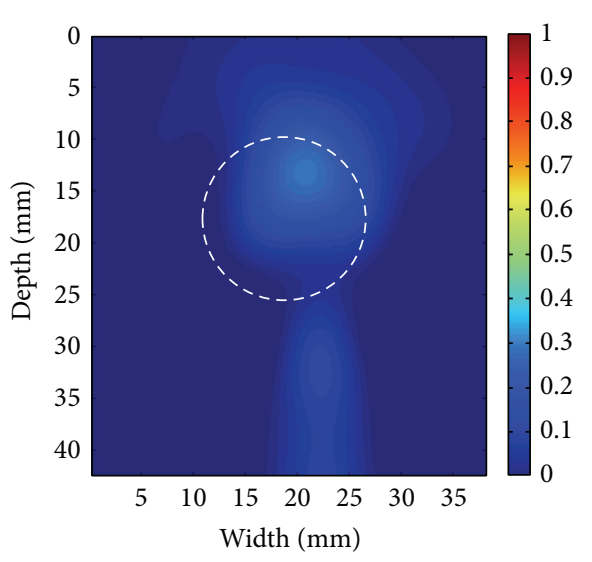

(g)

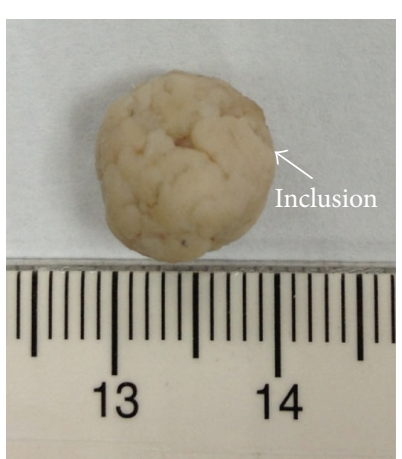

(h)

Figure 4: Electrode-displacement images obtained at different angles: (a) angle $=30^{\circ}$, (b) angle $=40^{\circ}$, (c) angle $=50^{\circ}$, (d) angle $=60^{\circ}$, (e) angle $=70^{\circ}$, (f) angle $=80^{\circ}$, and (g) angle $=90^{\circ}$; (h) embedded meatball in the inclusion phantom.

was completed. In this condition, the necrotic inclusion in the phantom produced a substantial axial displacement, when the RF electrode moved back and forth. The degree of adhesion of the RF electrode to the ablated tissue might further rely on the ablation temperature (or power), water content in tissues, the degree of necrosis, and the tissue type. In other words, how to identify "soft" and "hard" in electrode-displacement elastography in various clinical applications can be redefined.
4.3. Angle Effect on Electrode-Displacement Strain Imaging. In the following discussion, we attempt to clarify why a large angle between the ultrasound transducer and the RF electrode is unfavorable to forming electrode-displacement strain imaging. This can be explained using a sample discussion from mechanics. In conventional electrode-displacement strain imaging, the RF electrode is controlled by a onedimensional step motor to produce axial displacements, 


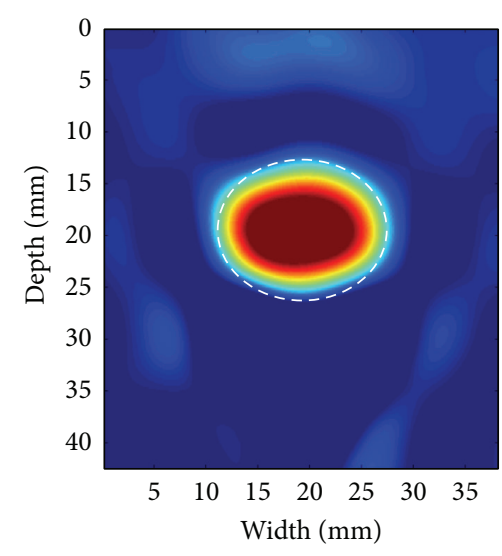

(a)

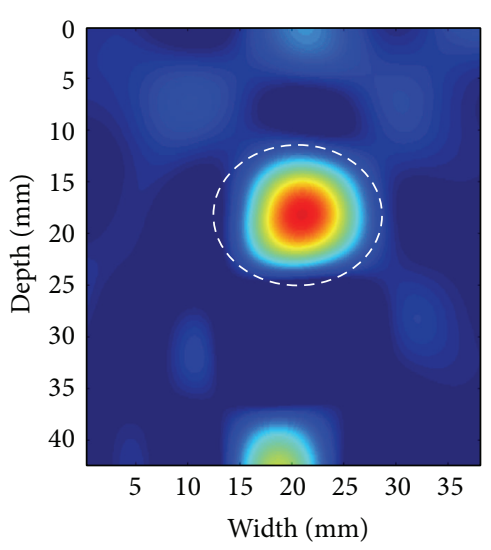

(d)

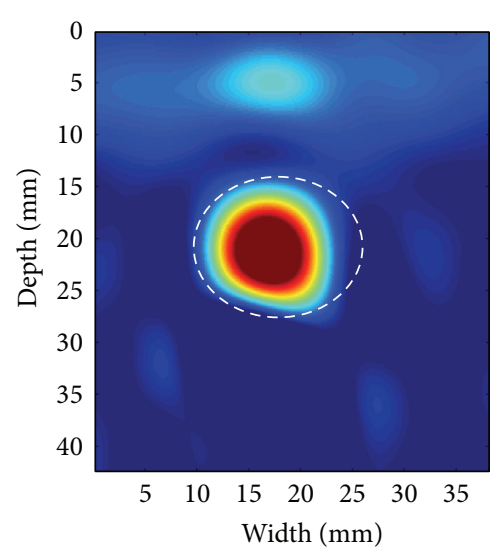

(b)

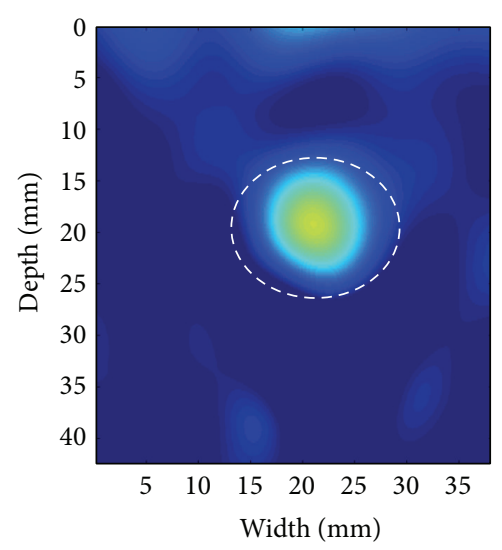

(e)

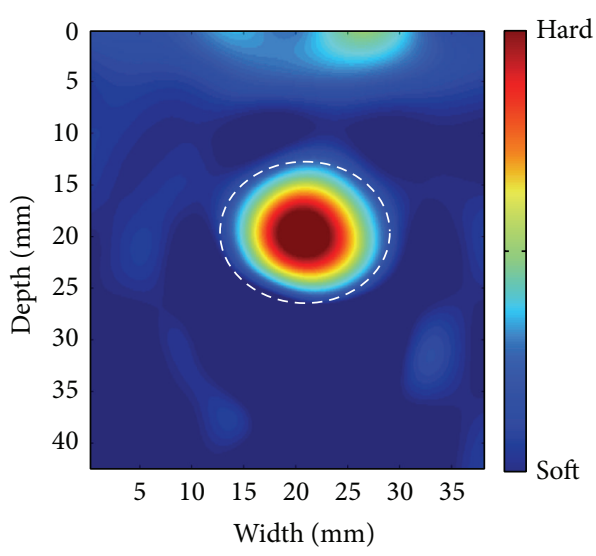

(c)

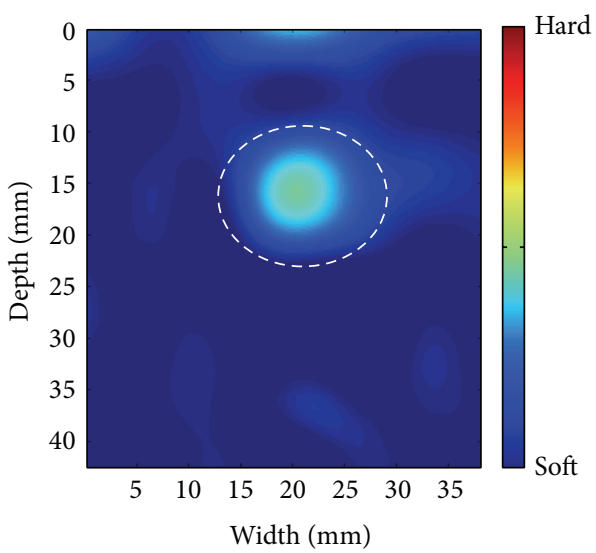

(f)

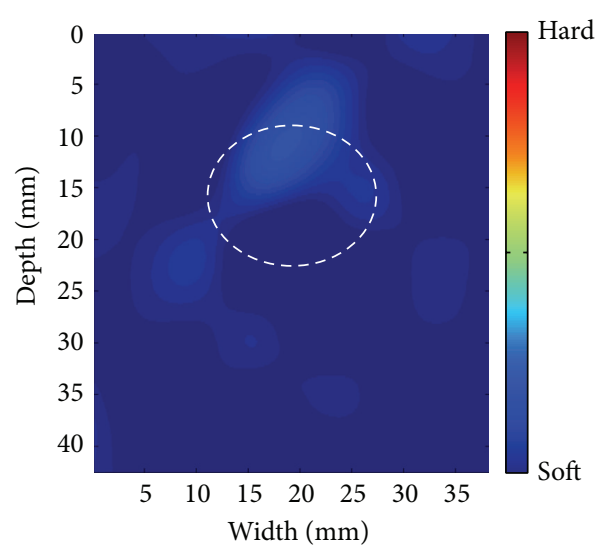

(g)

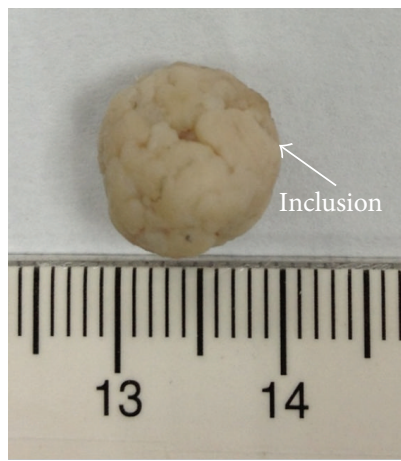

(h)

FIGURE 5: Electrode-displacement strain images obtained at different angles: (a) angle $=30^{\circ},(\mathrm{b})$ angle $=40^{\circ},(\mathrm{c})$ angle $=50^{\circ},(\mathrm{d})$ angle $=60^{\circ}$, (e) angle $=70^{\circ}$, (f) angle $=80^{\circ}$, and (g) angle $=90^{\circ}$; (h) embedded meatball in the inclusion phantom.

resulting in axial strain of tissues. When the angle between the ultrasound transducer and the RF electrode is small, the echo time-shift detected in the plane of ultrasound image can sensitively reflect tissue axial strain, producing a reliable electrode-displacement strain image. When the angle is large, the ultrasound image detects the lateral vector of the axial strain in the tissue. The echo time-shift corresponding to the lateral vector of the axial strain detected in the ultrasound image decreases with an increasing angle, rendering it difficulty to form the electrode-displacement strain image.

\subsection{Strengths and Limitations of Electrode-Displacement} Strain Imaging. Using ultrasound elastography to describe the necrosis region generated from RFA will be a trend in future clinical diagnosis. Both acoustic-radiation force impulse (ARFI) imaging and supersonic shear-wave imaging 


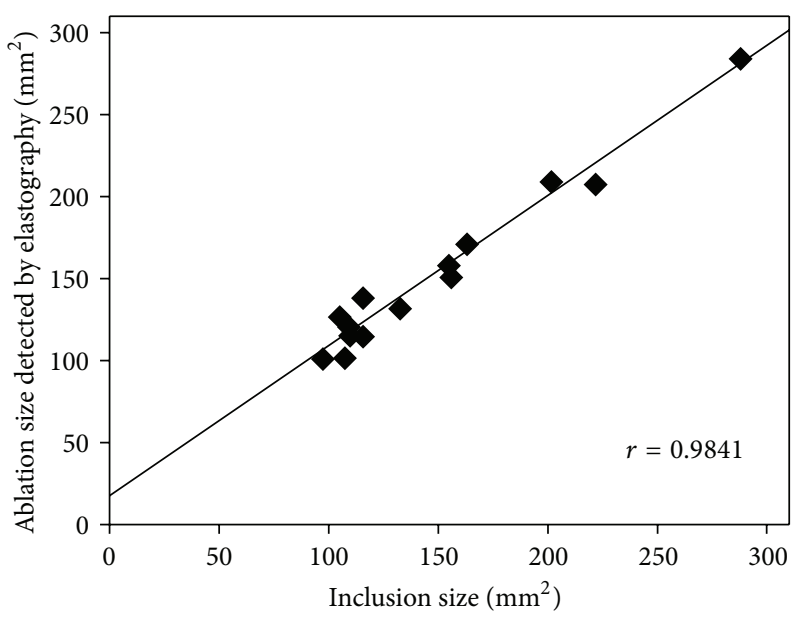

- Angle $=30^{\circ}$

— Linear regression

(a)

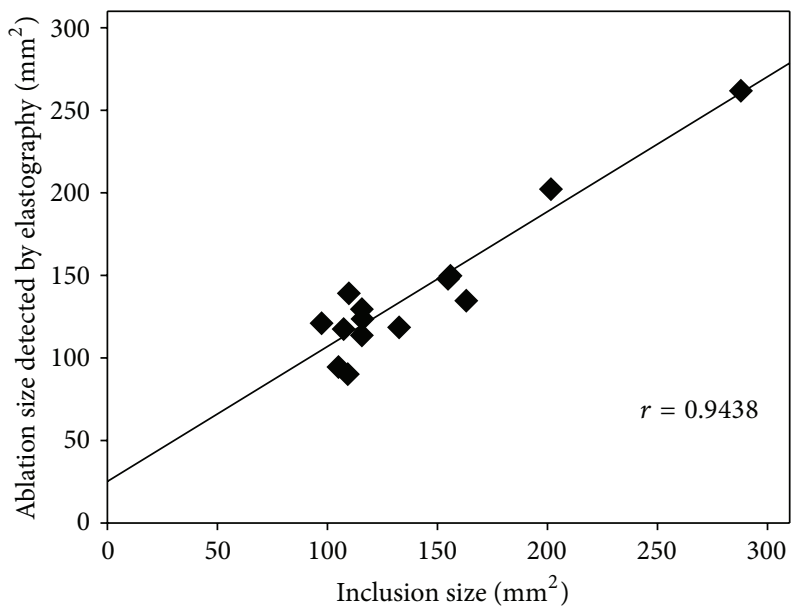

- Angle $=50^{\circ}$

— Linear regression

(c)

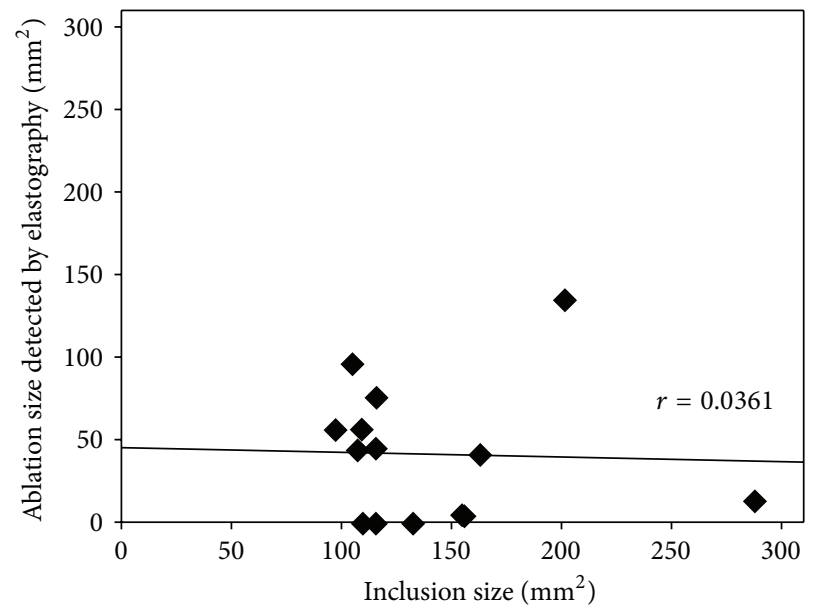

- Angle $=70^{\circ}$

_ Linear regression

(e)

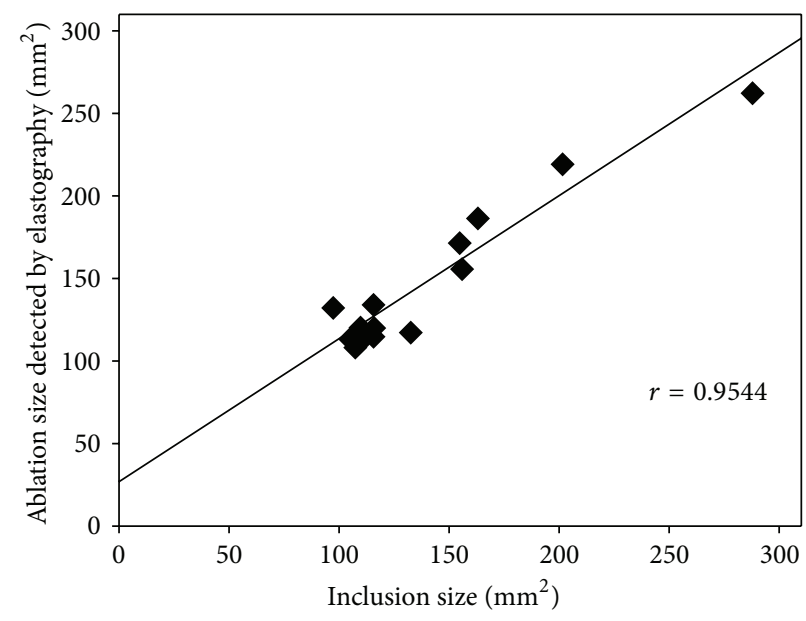

- Angle $=40^{\circ}$

— Linear regression

(b)

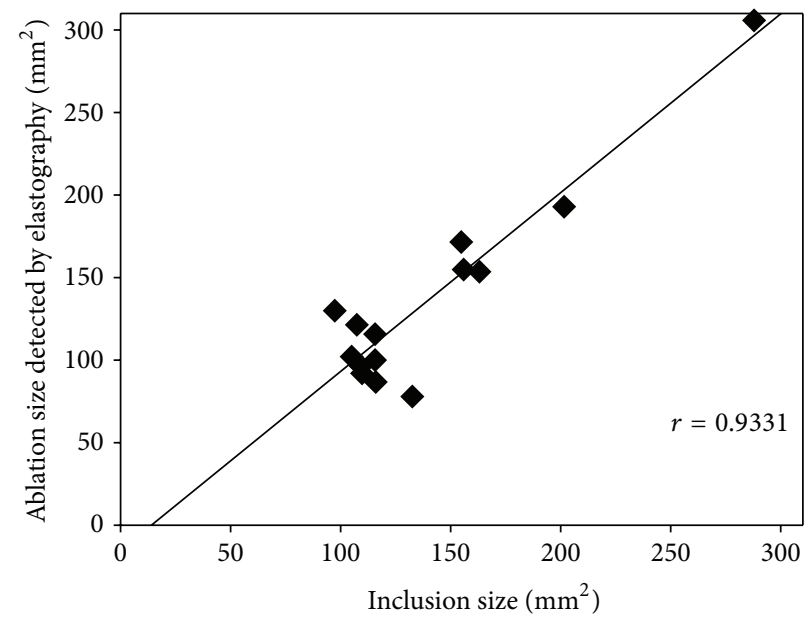

- Angle $=60^{\circ}$

_ Linear regression

(d)

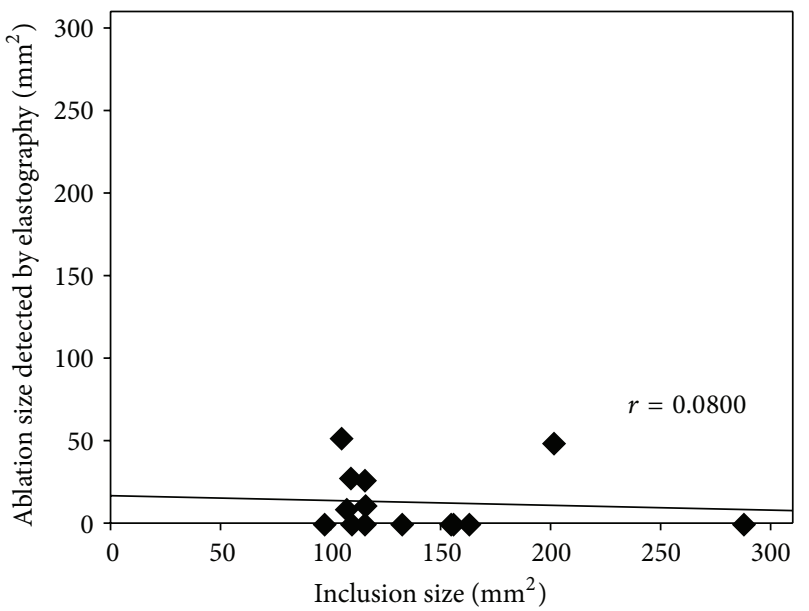

- Angle $=80^{\circ}$

— Linear regression

FIGURE 6: Continued. 


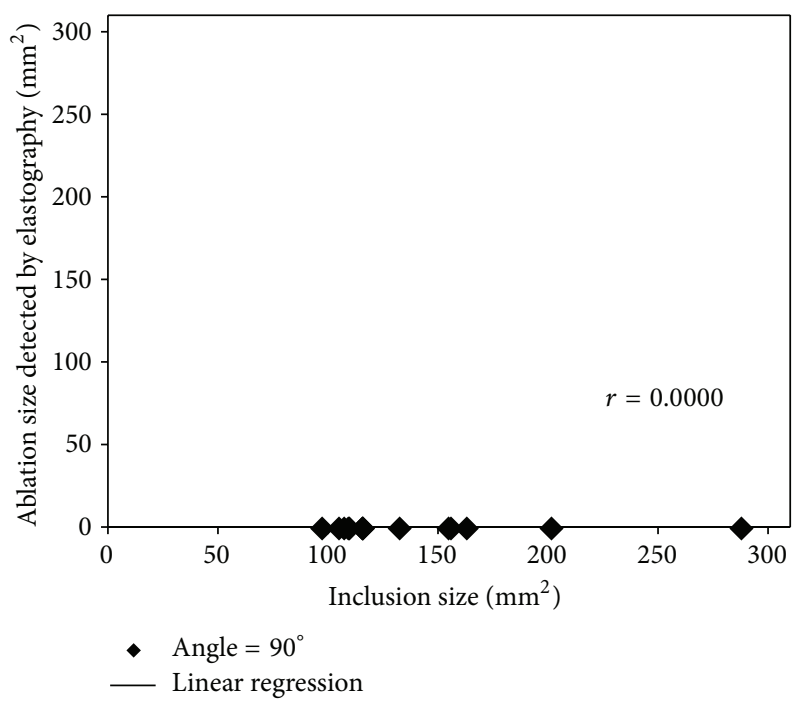

(g)

FIGURE 6: Comparisons between the meatball sizes and the ablation zones detected by electrode-displacement strain imaging $(n=14)$. (a) angle $=30^{\circ}$, (b) angle $=40^{\circ}$, (c) angle $=50^{\circ}$, (d) angle $=60^{\circ}$, (e) angle $=70^{\circ}$, (f) angle $=80^{\circ}$, and (g) angle $=90^{\circ}$. The correlation coefficient decreases with increasing the angle between the transducer and the RF electrode. No significant correlation was found when the angle was larger than $60^{\circ}$.

(SSI) are novel techniques for ultrasound elastography. A previous study used ARFI elastography and SSI for guiding and assessing thermal ablation $[35,36]$. ARFI elastography was developed to measure shear-wave velocity, which is related directly to tissue stiffness. The ARFI system measures shear-wave velocity by repeating push pulses and detecting pulses across a region of interest. Compared with ARFI, a shear-wave elastography system visualizes absolute tissue stiffness. SSI entails generating a remote radiation force by using focused ultrasonic beams (i.e., pushing beams). Each pushing beam generates a remote vibration that propagates a transient shear wave. Several pushing beams at increasing depths are transmitted to generate a quasiplane shear-wave front that propagates throughout the entire imaging area. After generating the shear wave, an ultrafast echographic imaging sequence is performed to acquire successive raw data at a high frame rate to estimate the shear-wave velocity and the shear modulus for imaging.

ARFI and SSI can provide superior boundary definition of structures relative to using conventional sonography alone. Using ARFI and SSI to monitor RFA does not require using the RF electrode to produce tissue strain (tissue strain is induced by pushing beams). Recent studies have also reported that using electrode perturbation can produce shear waves that are tracked at high frame rates for electrode vibration elastography [37-39]. However, ARFI and SSI have not yet been popularized in current commercial ultrasound systems. Therefore, electrode-displacement strain imaging remains strong and clinically crucial because it can be implemented using a conventional ultrasound B-scan system. Consequently, we suggested an appropriate angle for successfully forming electrode-displacement strain imaging as a crucial consideration in future clinical applications.
The threshold angle also implies a possible limitation when using the electrode-displacement strain image for monitoring RFA in vivo. That is, electrode-displacement strain imaging is not necessary to have the ability for monitoring the RFA of tumors at various locations and depths.

4.5. Suggestions for Future Studies. We have two suggestions for future work. (i) In the current study, electrodedisplacement strain imaging is based on one-dimensional cross-correlation analysis. Using a two-dimensional speckle tracking technique may improve the construction of electrode-displacement strain image using a large-angle arrangement. However, different algorithms may have different performances and efficiencies. Thus, the effects of various algorithms on electrode-displacement strain imaging need to be investigated and further compared. (ii) To mitigate the limitations of electrode-displacement strain imaging caused by the angle effect, we propose the possibility of combining electrode-displacement strain imaging and other imaging techniques by using the dualtransducer arrangement. One transducer can be responsible for electrode-displacement strain imaging at small angles to visualize the ablation zone, whereas the other transducer can be arranged at large angles to describe the ablation zone by implementing a certain imaging technique that is independent of the measurement angle (e.g., ultrasound Nakagami imaging proposed recently for monitoring RFA by modeling the envelope statistics [40]). In a clinical environment without advanced ultrasound systems, using a conventional grayscale ultrasound system combined with electrode-displacement strain imaging and other imaging techniques is a strategy for researchers to pursue. 


\section{Conclusion}

This study explores the effects of angle on using electrodedisplacement strain imaging to monitor RFA by performing experiments on inclusion phantoms. The primary contribution of this study is demonstrating that the angle required for successfully constructing electrode-displacement elastography should be smaller than $60^{\circ}$, allowing the ultrasound transducer to effectively reflect tissue axial strain caused by perturbations of the RF electrode. This experimental finding should be treated as a crucial consideration for future clinical applications and developments of electrode-displacement elastography.

\section{Conflict of Interests}

The authors declare that there is no conflict of interests regarding the publication of this paper.

\section{Acknowledgments}

This work was supported by the National Science Council of the Republic of China (Taiwan) under Grant no. NSC 102-2221-E-182-008 and the Chang Gung Memorial Hospital (Linkou, Taiwan) under Grant no. CMRPD1C0711. This work was also supported by the Chang Gung Medical Research Program (CMRP) for Proton Therapy Basic Research (Grant nos. CMRPD1C0661 and CMRPD1C0641).

\section{References}

[1] R. Siegel, D. Naishadham, and A. Jemal, "Cancer statistics, 2013," CA Cancer Journal for Clinicians, vol. 63, no. 1, pp. 11-30, 2013.

[2] P. Liang and Y. Wang, "Microwave ablation of hepatocellular carcinoma," Oncology, vol. 72, supplement 1, pp. 124-131, 2007.

[3] T. J. Vogl, K. Eichler, R. Straub et al., "Laser-induced thermotherapy of malignant liver tumors: general principals, equipment(s), procedure(s) - Side effects, complications and results," European Journal of Ultrasound, vol. 13, no. 2, pp. 117-127, 2001.

[4] T. Mala, "Cryoablation of liver tumours-a review of mechanisms, techniques and clinical outcome," Minimally Invasive Therapy and Allied Technologies, vol. 15, no. 1, pp. 9-17, 2006.

[5] H. B. El-Serag, J. A. Marrero, L. Rudolph, and K. R. Reddy, "Diagnosis and treatment of hepatocellular carcinoma," Gastroenterology, vol. 134, no. 6, pp. 1752-1763, 2008.

[6] R. Lencioni and L. Crocetti, "Radiofrequency ablation of liver cancer," Techniques in Vascular and Interventional Radiology, vol. 10, no. 1, pp. 38-46, 2007.

[7] S. N. Goldberg, "Radiofrequency tumor ablation: principles and techniques," European Journal of Ultrasound, vol. 13, no. 2, pp. 129-147, 2001.

[8] T. Varghese, U. Techavipoo, W. Liu et al., "Elastographic measurement of the area and volume of thermal lesions resulting from radiofrequency ablation: pathologic correlation," The American Journal of Roentgenology, vol. 181, no. 3, pp. 701-707, 2003.

[9] T. Varghese, J. A. Zagzebski, and F. T. Lee Jr., "Elastographic imaging of thermal lesions in the liver in vivo following radiofrequency ablation: Preliminary results," Ultrasound in Medicine and Biology, vol. 28, no. 11-12, pp. 1467-1473, 2002.
[10] T. Wu, J. P. Felmlee, J. F. Greenleaf et al., "Assessment of thermal tissue ablation with $\mathrm{mr}$ elastography," Magnetic Resonance in Medicine, vol. 45, no. 1, pp. 80-87, 2001.

[11] R. Righetti, F. Kallel, R. J. Stafford et al., "Elastographic characterization of HIFU-induced lesions in canine livers," Ultrasound in Medicine and Biology, vol. 25, no. 7, pp. 1099-1113, 1999.

[12] J. Ophir, I. Céspedes, H. Ponnekanti, Y. Yazdi, and X. Li, "Elastography: a quantitative method for imaging the elasticity of biological tissues," Ultrasonic Imaging, vol. 13, no. 2, pp. 111134, 1991

[13] T. Varghese, J. Ophir, E. Konofagou, F. Kallel, and R. Righetti, "Tradeoffs in elastographic imaging," Ultrasonic Imaging, vol. 23, no. 4, pp. 216-248, 2001.

[14] E. I. Cespedes, Elastography: imaging of biological tissue elasticity [Ph.D. thesis], University of Houston, 1993.

[15] M. Bertrand, J. Meunier, M. Doucet, and G. Ferland, "Ultrasonic biochemical strain gauge based on speckle tracking," in Proceedings of the IEEE Ultrasonics Symposium, pp. 859-863, October 1989.

[16] M. O’Donnell, A. R. Skovoroda, B. M. Shapo, and S. Y. Emelianov, "Internal displacement and strain imaging using ultrasonic speckle tracking," IEEE Transactions on Ultrasonics, Ferroelectrics, and Frequency Control, vol. 41, no. 3, pp. 314-325, 1994.

[17] M. O’Donnell, A. Skovoroda, and B. Shapo, "Measurement of arterial wall motion using fourier bases speckle tracking algorithms," Proceedings of IEEE Ultrasonics Symposium, vol. 2, pp. 1101-1104, 1991.

[18] J. Ophir, B. Garra, F. Kallel et al., "Elastographic imaging," Ultrasound in Medicine and Biology, vol. 26, pp. S23-S29, 2000.

[19] T. A. Krouskop, D. R. Dougherty, and F. S. Vinson, "A pulsed doppler ultrasonic system for making noninvasive measurements of the mechanical properties of soft tissue," Journal of Rehabilitation Research and Development, vol. 24, no. 2, pp. 18, 1987.

[20] K. J. Parker, S. R. Huang, R. A. Musulin, and R. M. Lerner, "Tissue response to mechanical vibrations for 'sonoelasticity imaging," Ultrasound in Medicine and Biology, vol. 16, no. 3, pp. 241-246, 1990.

[21] Y. Yamakoshi, J. Sato, and T. Sato, "Ultrasonic imaging of internal vibration of soft tissue under forced vibration," IEEE Transactions on Ultrasonics, Ferroelectrics, and Frequency Control, vol. 37, no. 2, pp. 45-53, 1990.

[22] E. Boctor, M. Deoliveira, M. Choti et al., "Ultrasound monitoring of tissue ablation via deformation model and shape priors," in Medical Image Computing and Computer-Assisted Intervention, vol. 4191 of Lecture Notes in Computer Science, pp. 405-412, Springer, Berlin, Germany, 2006.

[23] S. Bharat, T. Varghese, E. L. Madsen, and J. A. Zagzebski, "Radio-frequency ablation electrode displacement elastography: a phantom study," Medical Physics, vol. 35, no. 6, pp. 24322442, 2008.

[24] S. Bharat and T. Varghese, "Contrast-transfer improvement for electrode displacement elastography," Physics in Medicine and Biology, vol. 51, no. 24, article 6403, 2006.

[25] J. Jiang, T. Varghese, Q. Chen, T. J. Hall, and J. A. Zagzebski, "Finite element analysis of tissue deformation with a radiofrequency ablation electrode for strain imaging," IEEE Transactions on Ultrasonics, Ferroelectrics, and Frequency Control, vol. 54, no. 2, pp. 281-289, 2007. 
[26] K. Nightingale, M. S. Soo, R. Nightingale, and G. Trahey, "Acoustic radiation force impulse imaging: in vivo demonstration of clinical feasibility," Ultrasound in Medicine and Biology, vol. 28, no. 2, pp. 227-235, 2002.

[27] N. Rubert, S. Bharat, R. Dewall et al., "In vivo ultrasound electrode displacement strain imaging," in Proceedings of the IEEE International Ultrasonics Symposium (IUS '09), pp. 131134, September 2009.

[28] N. Rubert, S. Bharat, R. J. DeWall et al., "Electrode displacement strain imaging of thermally-ablated liver tissue in an in vivo animal model," Medical Physics, vol. 37, no. 3, pp. 1075-1082, 2010.

[29] S. Bharat, U. Techavipoo, M. Z. Kiss, W. Liu, and T. Varghese, "Monitoring stiffness changes in lesions after radiofrequency ablation at different temperatures and durations of ablation," Ultrasound in Medicine and Biology, vol. 31, no. 3, pp. 415-422, 2005.

[30] S. Bharat, T. G. Fisher, T. Varghese et al., “Three-dimensional electrode displacement elastography using the siemens C7F2 four sight four-dimensional ultrasound transducer," Ultrasound in Medicine and Biology, vol. 34, no. 8, pp. 1307-1316, 2008.

[31] E. Biagi, L. Breschi, and L. Masotti, "Transient subharmonic and ultraharmonic acoustic emission during dissolution of free gas bubbles," IEEE Transactions on Ultrasonics, Ferroelectrics, and Frequency Control, vol. 52, no. 6, pp. 1050-1056, 2005.

[32] T. G. Leighton, The Acoustic Bubbles, Academic Press, San Diego, Calif, USA, 1997.

[33] T. D. Mast, D. P. Pucke, S. E. Subramanian, W. J. Bowlus, S. M. Rudich, and J. F. Buell, "Ultrasound monitoring of in vitro radio frequency ablation by echo decorrelation imaging," Journal of Ultrasound in Medicine, vol. 27, no. 12, pp. 1685-1697, 2008.

[34] I. Winkler and D. Adam, "Monitoring radio-frequency thermal ablation with ultrasound by low frequency acoustic emissionsin vitro and in vivo study," Ultrasound in Medicine and Biology, vol. 37, no. 5, pp. 755-767, 2011.

[35] Y. Takuma, K. Nouso, Y. Morimoto et al., "Measurement of spleen stiffness by acoustic radiation force impulse imaging identifies cirrhotic patients with esophageal varices," Gastroenterology, vol. 144, no. 1, pp. 92-101, 2013.

[36] R. Ternifi, J. L. Gennisson, M. Tanter et al., "Effects of storage temperature on the mechanical properties of porcine kidney estimated using shear wave elastography," Journal of the Mechanical Behavior of Biomedical Materials, vol. 28, pp. 86-93, 2013.

[37] S. Bharat and T. Varghese, "Radiofrequency electrode vibrationinduced shear wave imaging for tissue modulus estimation: a simulation study," Journal of the Acoustical Society of America, vol. 128, no. 4, pp. 1582-1585, 2010.

[38] R. J. Dewall, T. Varghese, and E. L. Madsen, "Shear wave velocity imaging using transient electrode perturbation: phantom and ex vivo validation," IEEE Transactions on Medical Imaging, vol. 30, no. 3, pp. 666-678, 2011.

[39] R. J. Dewall, T. Varghese, and C. L. Brace, "Visualizing ex vivo radiofrequency and microwave ablation zones using electrode vibration elastography," Medical Physics, vol. 39, no. 11, pp. 66926700, 2012.

[40] C. Y. Wang, X. Geng, T. S. Yeh, H. L. Liu, and P. H. Tsui, "Monitoring radiofrequency ablation with ultrasound Nakagami imaging," Medical Physics, vol. 40, no. 7, Article ID 072901, 2013. 


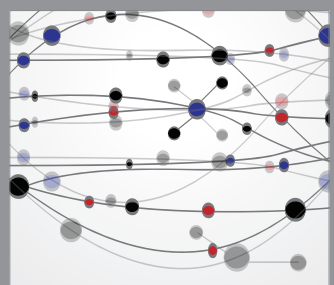

The Scientific World Journal
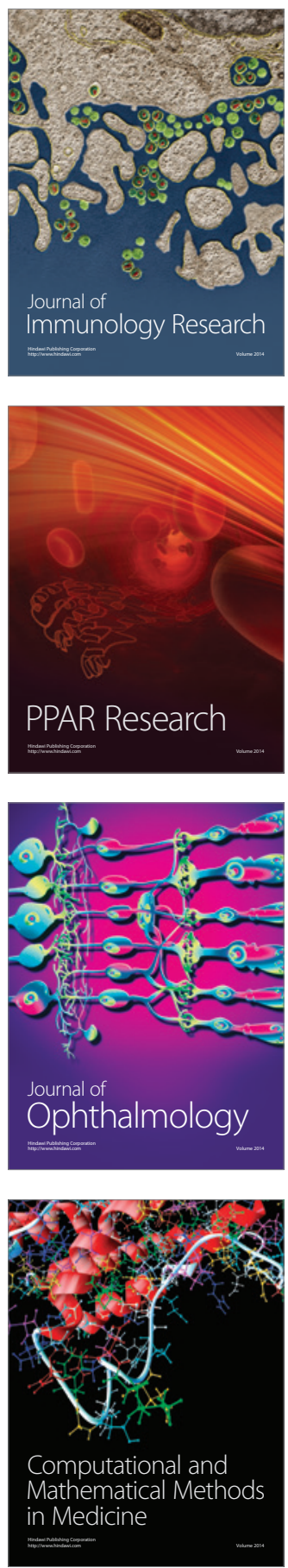

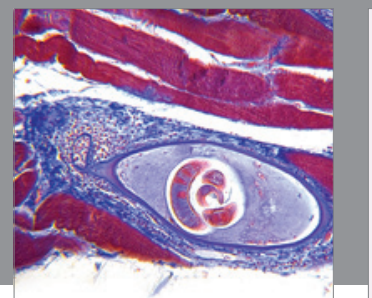

Gastroenterology

Research and Practice
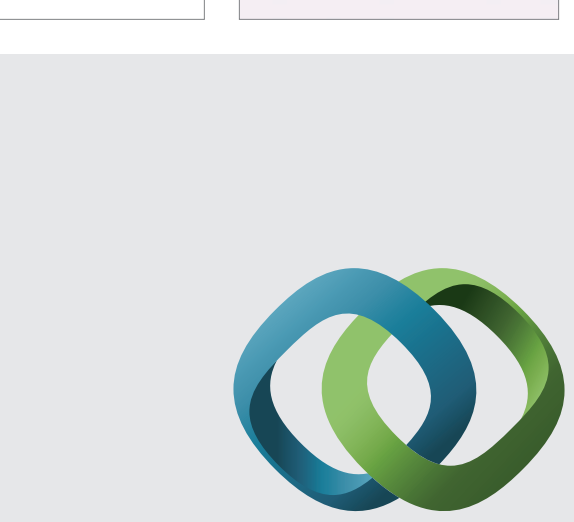

\section{Hindawi}

Submit your manuscripts at

http://www.hindawi.com
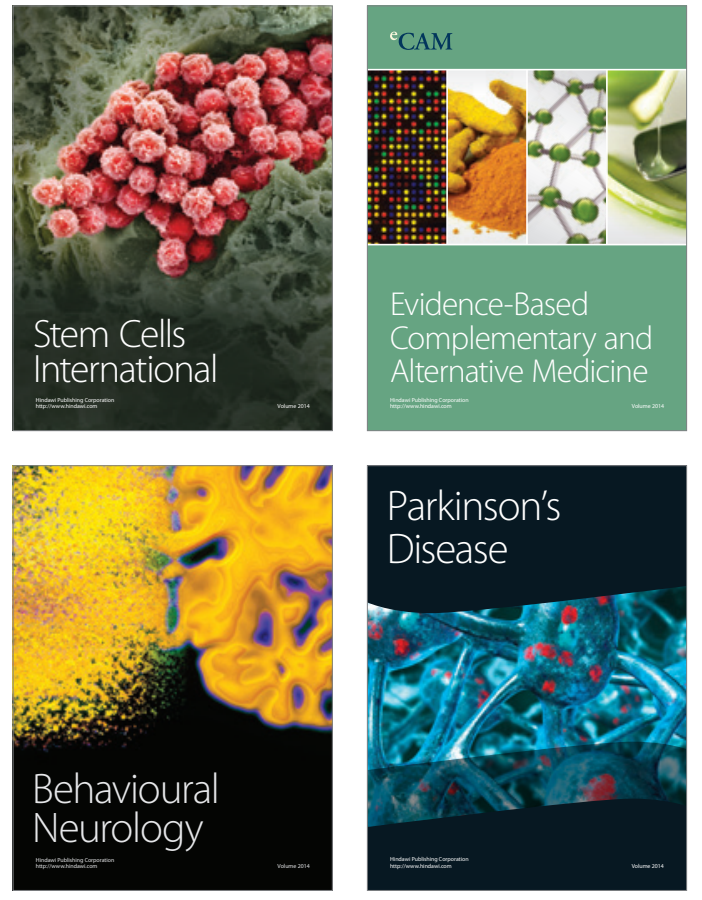
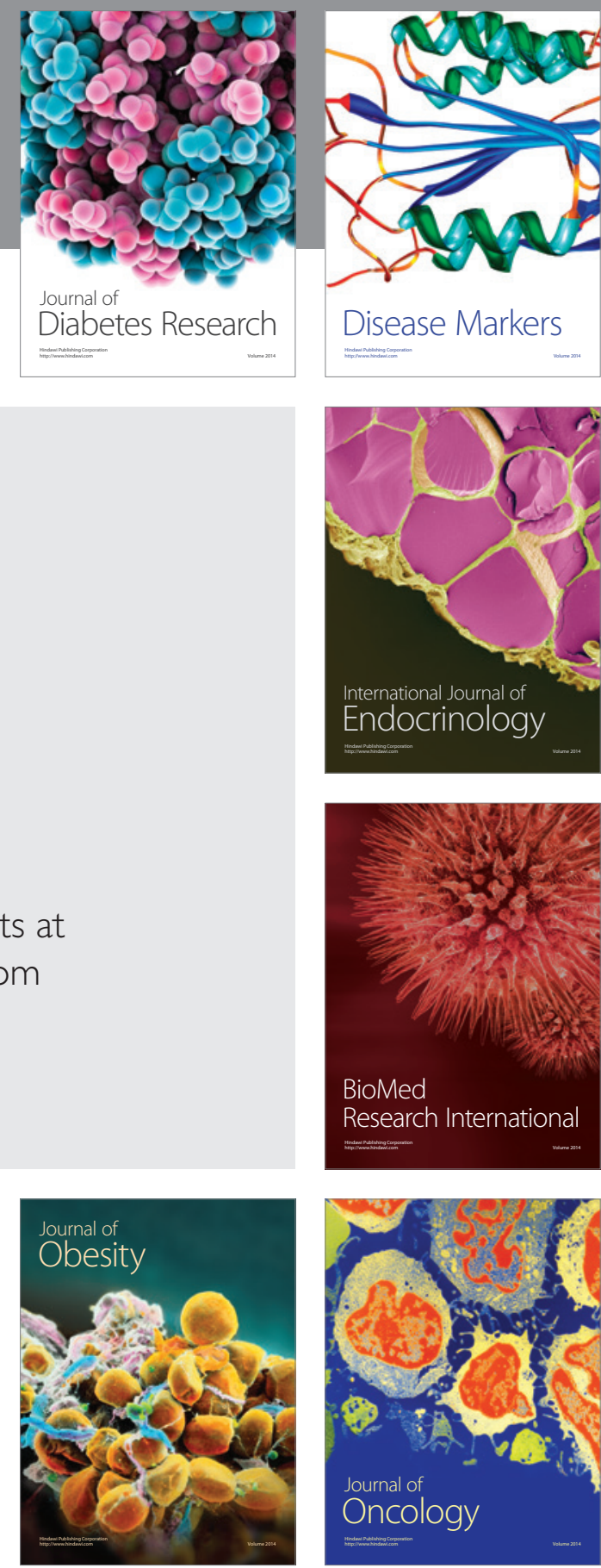

Disease Markers
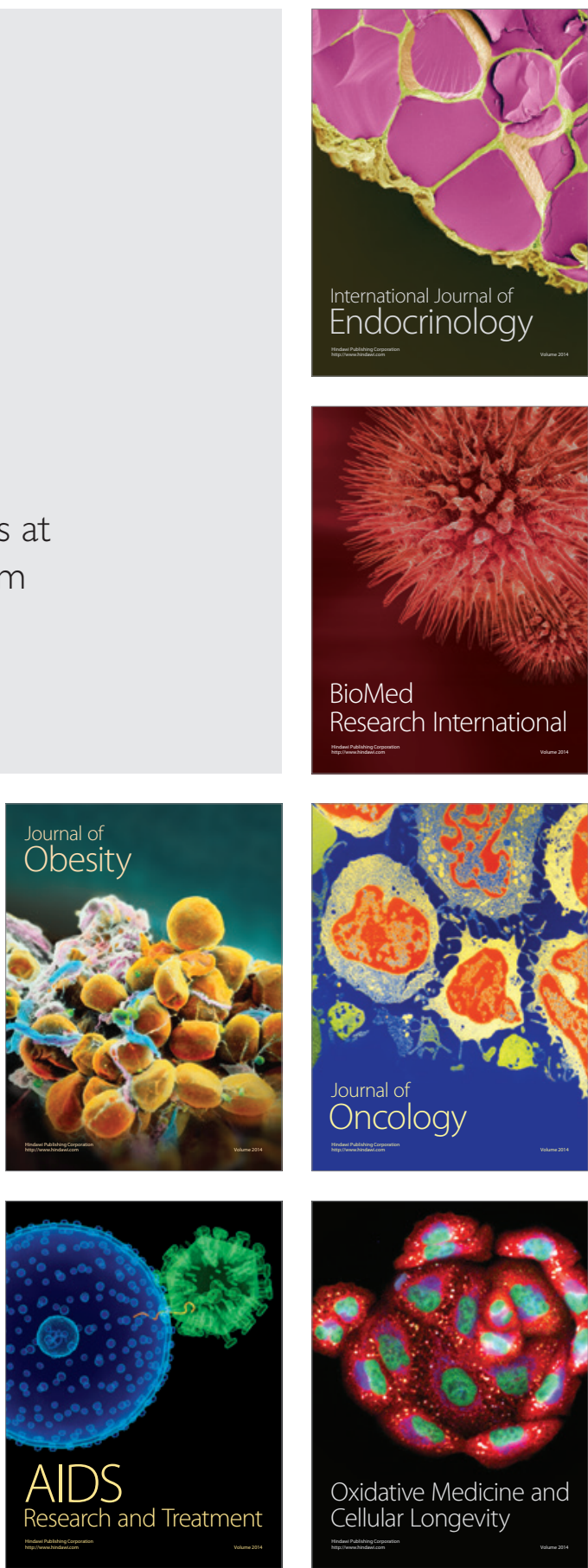